\title{
Holistic Appraisal of Modeling Installed Antennas for Aerospace Applications
}

\author{
A. Vukovic, P. Sewell, T. M. Benson
}

\begin{abstract}
This paper uses the Unstructured Transmission Line Modeling Method to investigate near field interactions between a broadband microwave antenna and a platform that arise as a result of antenna installation. The antenna, feed line and the platform are represented by a common meshed model and simulated using a single time-domain numerical method. This paper aims to establish guidelines on how to achieve high accuracy when modeling both the near and far fields of an antenna whilst at the same time prioritizing computational resources. By isolating critical features such as the feed line and selected fine details of the antenna geometry the paper assesses how accurately these fine features need to be described in the model and how they affect the return loss and far field pattern of the antenna. The size of the platform, is varied from small to medium size (up to 10 wavelengths) and its impact on the antenna performance is assessed. Finally, the conclusions of the study are applied to an example of an antenna installed in the leading edge of an aircraft wing, with and without, a protective radome cover.
\end{abstract}

Index Terms-Numerical analysis, broadband antennas, aircraft antennas.

\section{INTRODUCTION}

There is substantial interest in modeling antennas in their practical setting given that an antenna's performance can be significantly affected by its surroundings [1-14]. However, this is a computationally challenging task requiring accurate representation of both antenna and platform geometries which may differ in size by several orders of magnitude. In particular, antennas for many applications, such as $5 \mathrm{G}$ mobile and radar, come in the form of large arrays, where both mutual coupling between antenna elements and the array and the platform need to be accurately accounted for [15]. Furthermore, the increased presence of complex inhomogeneous structures and non-linear and anisotropic materials further complicates simulation and proves awkward for methods that are usually optimized for perfectly conducting bodies such as the Method of Moments (MoM).

The multiscale nature of such problems leads to high computational demands, regardless of the method used. For this reason, simplified approaches are still routinely employed, either approximating aspects of the geometry and/or materials or else the physical coupling mechanisms between the antenna and its platform.

The first simplified approach is illustrated by asymptotic methods such as ray tracing [7] which are indeed viable approaches for solving very large problems but are typically limited to PEC structures and surfaces that can be described by approximate reflection coefficients and as such, lack accuracy when considering antennas with complex features [16].

The second simplified approach which is commonly used is to replace the intricate detail of antenna geometries by simpler equivalents. These may be obtained by use of the reciprocity theorem $[3,17,18]$, an equivalent dipole model [4], or by using domain decomposition techniques with suitable solvers employed in each domain $[5,6]$. This approach to installed antennas has been adopted in conjunction with a range of particular methods, both asymptotic and full wave. The obvious disadvantage is either partial or complete

Manuscript received August 30, 2017. The authors are with the George Green Institute for Electromagnetics research, School of Electrical and Electronic Engineering, The University of Nottingham, Nottingham University Park, NG7 2RD, Nottingham, UK. (email: ana.vukovic@nottingham.ac.uk) Digital Object Identifier 10.1109/TAP.2016.xxx decoupling of an antenna from its practical setting and hence the nearfield interactions between antenna and the platform are not properly accounted for.

When considering practically large and complex platforms one common practice is to scale down the antenna's operating frequency [19] and thus increase the overall antenna dimensions or alternatively scale up the antenna's operating frequency and scale down the overall size of the platform [1]. The former approach works well at lower frequencies employing quasi-static analysis but will not capture any resonant interactions between the antenna and the platform. In contrast, the latter approach can over-emphasize or introduce resonant interactions that do not physically exist.

An excellent overview of a range of methods currently in use, discussing their advantages and disadvantages for modeling antennas in their practical setting, is given in [8].

At this point it is also important to recognize that installed antennas may be present within a larger scale simulation whose primary aim is not the characterization of the antenna. For example, lightning zoning and electromagnetic compatibility studies for aircraft certification [20, 21]. In such cases, it is attractive to be able to capture the antenna's installed behavior using the same flexible method employed for the overall context without undue need for special treatments for reasons of efficiency. The relevance to this work is that it demands a holistic view when selecting a modeling method.

It might be suggested that inexorable increases in computer power will inevitably permit full-wave algorithms such as Finite Difference Time Domain Methods (FDTD) to be straightforwardly used for installed antenna modeling. However, experience shows that computational resources are never adequate to fulfill the modeling ambitions of the innovative designer. There are a number of candidate full wave numerical methods that are actively in use, all benefitting from improved computing power: The Method of Moments (MoM), [22], Finite Element Time Doman (FETD) method [23], Finite Difference Time Domain Method (FDTD) [24] and Transmission Line Modeling (TLM), [25, 26].

The accuracy of all full-wave methods that spatially discretize the geometry is fundamentally dictated by the sampling density. Text book figures of $\lambda / 10$, where $\lambda$ is the operating frequency, are well known, [22-26] but of course derive from representing plane wave propagation in free space. Capturing rapidly varying static field behavior and physically important geometrical subtleties usually demands significantly better than this. Nevertheless, illustrations from the literature support the argument that increased computational power and parallel processing have brought configurations such as models of real-size aircraft within the reach of powerful computer platforms.

For example, the FDTD method has recently been used to model a dipole antenna installed on the nose of an aircraft using a uniform cubic mesh of size $\lambda / 23$ [2]. Similarly, [2] reported the modeling of an electrically small patch antenna on the roof of a car using a mesh of $\lambda / 114$ around the antenna which is graded up to $\lambda / 20$ for the rest of the problem space. In both cases such small cubic meshes were needed in order to ensure the stability of the FDTD Method and to reduce the dispersion and numerical errors caused by the sugar cube approximation of the geometry [8].

Clearly, physically informed resource management is critically important. So, as it is well known that the structure immediately 
surrounding the antenna has the greatest impact on its performance in contrast to more distant features, examples such as $[1,2]$ exploit nonuniform sampling. For example, in [1], sampling densities of $\lambda / 8$ for the platform sufficiently far from the antenna, $\lambda / 16$ for the near field region of the antenna using a frequency domain Multi-Level Fast Monopole (MLFMM) method.

Notwithstanding the differences between methods, for example, FDTD and MLFMM, in this work it is argued that (a) current resources do permit full wave methods to be deployed to provide better predictions for installed antenna performance; (b) The modeler must carefully manage the use of resources but to do so, (c) must understand the relative impact of compromising and/or enhancing different physical aspects of the problem and its geometrical representation. By means of an investigation of a representative case study, the key issues are identified and discussed in comparison with previous work and general guidelines are proposed.

To undertake this study we apply, for the first time, the Transmission Line Modeling method using unstructured meshes (UTLM) [27-35] to the problem of installed antennas. Selection of this method proceeds from the well-known fact that unstructured meshes require notably fewer sample points to capture curved and multiscale geometries and if used intelligently in a hybrid fashion with Cartesian grids to model large empty space regions, this can be achieved without unduly compromising computational efficiency or with the complexity of bespoke sub-gridding techniques. Unstructured meshes are routinely used with FE methods [23] and have been developed for FDTD [36-38]. However, the TLM framework offers distinctly valuable features. First, there is no need to approximate the theoretical basis of TLM algorithm, using for example mass lumping, in order to obtain an explicit time stepping algorithm. Second, compared to the FDTD method, the electric and magnetic field samples are co-located in time and space. Third and most importantly, the stability of a UTLM algorithm is provable a-priori on a cell-by-cell basis without resorting to estimators such as the Courant condition. For large scale simulations this is a critical advantage as late time instability has never been observed with TLM. Being a time-domain method, UTLM easily permits modeling of both linear, dispersive and nonlinear materials. Finally, recent work has accounted for both electric and magnetic material losses [28], and the presence of carbon fiber panels embedded as thin film layers between the mesh cells [29].

This paper analyses 3 different antenna configurations. First, an antenna on an electrically small platform. This is typical of an antenna being modeled in isolation in order to converge the model with respect to sampling density and other simulation parameters of the antenna in order to obtain accurate predictions of overall performance. Performance is assessed in terms of both the return loss and the radiation pattern which permit assessment of sensitivity to both near and far field representations. The study is then extended to modeling a broadband antenna placed on an electrically large flat ground plane in order to investigate how the presence of the platform affects the overall antenna performance. Finally, the guidelines deduced from the previous analyses are used to study the performance of an antenna installed in the leading edge on an aircraft wing.

The paper is structured as follows: Section II summarizes the main characteristics of the UTLM method. Section III outlines the UTLM model of a broadband Vivaldi antenna on an electrically small platform and explores the meshing requirements in order to achieve good accuracy for both near and far field parameters. Specific attention is given to the geometrical description of the antenna feed and the use of mesh refinement in the near field region. Section III further examines how the size of the platform affects the antenna's radiation pattern and return loss and investigates whether reductions in the computational box size can be usefully made when modeling antennas installed on electrically large platforms. Section IV predicts the in-situ performance of a Vivaldi antenna installed in the leading edge of the aircraft wing and investigates how the presence of the wing and then a radome cover affects the radiation parameters of the antenna. Section V summarizes the main conclusions of the paper.

\section{THE UTLM METHOD}

In this section the main algorithm of the UTLM method and the associated mesh generation requirements are overviewed. It is emphasized that the focus of the paper is not to introduce the method, rather its use for the first time, exploring the choice of parameters, for application to installed antennas. Whilst not as well known as FDTD, the first 3D UTLM approach was initially presented in 2005, [27] and has been continuously validated and developed since then in order to yield an industrial strength capability. Particularly significant steps are highlighted as [30] and [34] as these have provided the robustness to scale and complexity demanded by installed antenna applications. Supporting evidence for the accuracy and multiscale capability of UTLM as well as its industrial deployment can be found in [27-35] which have primarily concerned EMC problems to date.

The UTLM method decomposes the problem space into nonoverlapping discrete tetrahedral cells forming a Delaunay mesh [39], the tangential electric and magnetic fields being sampled on the faces of each cell. Coupling a local harmonic field expansion within each cell with the requirement for tangential field continuity between cells results in a time domain algorithm that updates the field samples as time evolves [27]. Characteristic of all TLM approaches, this local field expansion and imposition of continuity mimic a scatter-connect process occurring in an analogous transmission line network.

The choice of a time step for the UTLM algorithm, $\Delta_{t}$, is dependent upon the quality of the mesh and its suitability is assessed using a canonical scattering problem for each cell considered in isolation. Note that the UTLM implementation does not become unstable with larger time steps, rather loses accuracy [27, 30]. Typically, very small cells, adjacent cells whose scattering centers approach each other, or quasi-flat sliver like cells will demand the use of a small time step for accuracy. Unfortunately, all of these possibilities are routinely encountered in many tetrahedral meshes. Initially, this presented a serious limitation for the choice of time step resulting in a computationally inefficient algorithm. The breakthrough for the UTLM method was to pre-process groups of cells into clusters forming larger scattering entities, [30]. The structure of these clusters easily permits separation of the physically meaningful field responses from those identifiable as sampling noise. The latter, which depend upon the precise local layout of the mesh sampling, are those demanding small time step values. Hence, this separation conveniently facilitates selection of a time step that accurately captures the physics without being constrained by a stability requirement to be impractically small. In practice, this critical clustering phase is user controlled by setting a threshold distance and groups of adjacent cells whose centers are closer than this value are automatically clustered into larger scattering entities. A point that shall be discussed more fully in the context of the results, is that specifying the threshold distance is strongly related to declaring a maximum frequency for which wave, as opposed to quasi-static, behavior is of interest.

It is commented that it is the requirement that the mesh be Delaunay that facilitates the UTLM approach and whilst this precludes use of, for example, advancing front meshes, this has not proved practically inconvenient. However, experienced modelers of industrial scale 
problems are well aware that successful simulations demand more than just a robust implementation of an accurate EM algorithm. Starting from real CAD data, generating a tetrahedral mesh by any means is a non-trivial task. As recently described in [34], geometrical features that might have little physical impact overall can, nevertheless, introduce substantial complications for subsequent simulation. It is naïve to consider meshing as an available black box activity that is undertaken without understanding its impact on accuracy, stability, memory and run time demands of the simulation algorithm. Therefore, a powerful complexity reduction approach has recently been developed to substantially extended the scope for clustering to cases when the meshing of practical CAD data yields regions comprising 1000s of small cells with little physical basis, [34].

The interrelation between the performance of the simulation algorithm and the mesh generation process is not often discussed in detail and this proves a critical consideration for successful modeling. For this reason, we highlight this aspect of the current study as a substantial contribution of this work.

When undertaking unstructured mesh generation, the user typically has two degrees of freedom. First, a measure of the uniformity of the 3D shape of the tetrahedral cells. This is often expressed by the mesh quality parameter, Q, which is defined as the ratio between the circumradius to the minimum edge length of each cell [39] with a related $2 \mathrm{D}$ measure of the quality of the triangular faces of the tetrahedral cells defined in the same manner. These two measures respectively characterize the uniformity of the field sampling in 3D space and the representation of the geometrical surfaces. Typically, a high quality Delaunay mesh has a value of $\mathrm{Q}=2$ with many practical geometries producing a good quality mesh with $3<\mathrm{Q}<6$. Imposing this requirement for quality will produce a mesh of well-shaped elements that is naturally denser around small and curved features of the model than in large empty regions of space. Moreover, good quality meshes produce less sampling noise in the subsequent simulations.

The second degree of freedom available to the modeler is to further control this good quality meshing by setting localized targets for the maximum cell volumes. This can initially be guided by simple knowledge of the operating wavelengths and then, more valuably, designer understanding of where rapid field variations are likely to occur due to quasi-static behavior. Throughout, the designer must consider the sensitivity to crucial aspects such as the feed points.

Generally, the disadvantage of using purely tetrahedral meshes is that they are less computationally efficient than their Cartesian counterparts. Therefore, in practice and throughout this paper, selective use of tetrahedral meshing is hybridized with cubic meshes to discretize the empty space regions. Such hybridization does not require special treatment in either mesh generation or subsequent UTLM time stepping. Seeding the mesh with sample points laid out in a grid yields UTLM computational cell clusters which are cuboidal cells and whose time stepping responses are the known special Cartesian cases which is very attractive for maximizing computationally efficiency for parallel, cache-conscious codes.

All meshes used in this study are obtained using our in-house Delaunay Mesher software [40] with a single pass used to holistically discretize the complete problem.

\section{Antenna Models}

In this section a broadband Vivaldi antenna is considered in 3 different settings namely, on an electrically small platform which is typical of an antenna being modeled in isolation, on an electrically large flat ground plane, and finally, installed in the leading edge of an aircraft wing. Modeling the antenna in isolation enables exploration of different meshing strategies in order to converge the results for both the near field parameter $S_{11}$ and far field radiation patterns. The sensitivity of the antenna parameters to the accuracy of the geometrical description of the coaxial feed line and the computational box size is also investigated with the aim of achieving the most computationally efficient simulations without unduly compromising accuracy. This analysis is then extended by placing the antenna on an electrically large ground plane and assessing the impact of the installation on the antenna performance together with an assessment of different meshing densities. Finally, the conclusions of the above analysis are applied to modeling the broadband Vivaldi antenna installed in the leading edge of the of the aircraft wing, with and without a radome cover.

\section{A Antenna Model}

The geometry of the Vivaldi antenna mounted on a conducting platform shown in Fig. 1 is taken from [19]. The slot line is printed on a dielectric substrate of $\varepsilon_{\mathrm{r}}=3$ and is exponentially flared to provide a smooth impedance transition from the coaxial feed to free space. The half width of the slot line, $w$, varies as $w(z)=0.25 e^{0.0797 z}$ and reaches $20 \mathrm{~mm}$ at the open mouth. The radius of the balun, realized as an etched circuit in the flared metallic region, is $2.5 \mathrm{~mm}$. The height and thickness of the dielectric slab are $55 \mathrm{~mm}$ and $1.5 \mathrm{~mm}$ respectively. The coaxial feed has core and outer diameters of $2 \mathrm{~mm}$ and $6 \mathrm{~mm}$ and a dielectric constant of $\varepsilon_{\mathrm{r}}=1$. The overall dimensions of the ground plane and the substrate are $70 \mathrm{~mm} \times 60 \mathrm{~mm}$ and $55 \mathrm{~mm} \times 40 \mathrm{~mm}$ respectively as shown in Fig. 1a,b). The antenna is excited with the TEM mode of the coaxial feed modulated by a time domain pulse with $3 \mathrm{~dB}$ frequencies of $1.8 \mathrm{GHz}$ and $4.6 \mathrm{GHz}$. A $4 \mathrm{~mm}$ length of the coaxial cable is placed inside the ground plane which is of $5 \mathrm{~mm}$ thickness. The antenna is enclosed by a Huygens surface placed at $\lambda / 2$ from the structure making the overall dimensions of the computational box $1.7 \lambda \times 1.6 \lambda \times 2.05 \lambda$, where $\lambda$ is the free space wavelength at the antenna's resonant frequency of $3 \mathrm{GHz}$.

The antenna is excited using the fundamental coaxial mode which is obtained as an eigen-solution of the discretized two-dimensional cross section of the cable [33]. It is important to emphasize that the same mesh that is used to discretize the whole antenna model is also

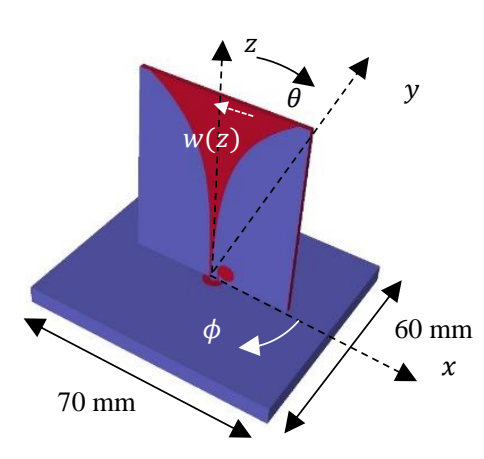

(a)

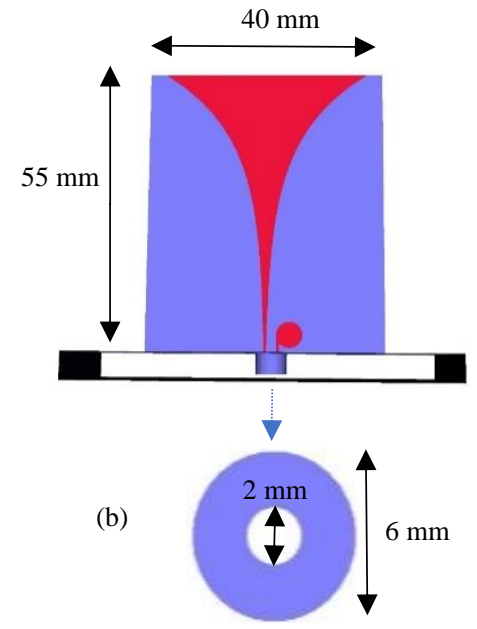

Fig. 1. a) Vivaldi antenna on a ground plane showing tapered slot line and the balun; b) front view and detail inside the ground plane and showing the crosssection of the coaxial feed cable. 
used to characterize the excitation mode which eliminates nonphysical reflections which would otherwise be generated by either applying the theoretical solution or using an independent mode solver that may discretize the cable in a different manner. The method for obtaining the excitation, which is fully described in [33], has second order convergence with mesh size for the modal wave impedance and effective relative permittivity and is able to provide good approximations to the true mode profile even when the mesh size or the feed's geometrical descriptions are crude. Both the discretization and geometrical description of the cable will have an effect on the accuracy of the performance and are hence investigated in this paper.

The first mesh selected for the model, shown in Fig. 2, is a good quality $(\mathrm{Q}=2)$ mesh naively allowing the sizing to be dictated solely by the requirement for the mesher to capture the geometry. This case is included to illustrate that the modeler must certainly impose more control than this. Whilst the fine detail of the antenna geometry is well resolved, Fig. 2a, the mesh is crude in the free space region, Fig. $2 b$. A simple first improvement is shown in Fig. 3 where a hybrid mesh, combining a cubic mesh of unit cell size of $\lambda / 20$ with the tetrahedral mesh, achieves both fine resolution of the antenna geometry and a uniform sampling of the free space region around the structure.

The case for use of this hybrid mesh may be summarized as relying on a good quality tetrahedral mesh to capture the geometry yet ensuring adequate wavelength resolution with the cubic mesh. However, as yet, it does not specifically target predictable rapid varying quasi-static field regions.

The convergence of the antenna field patterns with mesh size is now specifically investigated with hybrid meshes embedding cubic meshes of unit cell size of $\lambda / 5, \lambda / 10, \lambda / 15, \lambda / 20$ and $\lambda / 40$ which correspond to cubic cells of size $20 \mathrm{~mm}, 10 \mathrm{~mm}, 7 \mathrm{~mm}, 5 \mathrm{~mm}$ and $2.5 \mathrm{~mm}$ respectively.

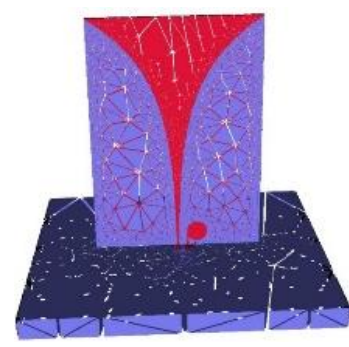

(a)

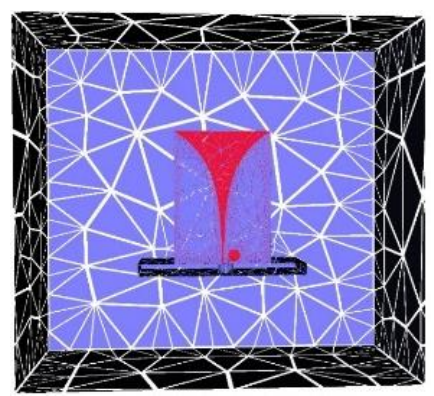

(b)
Fig. 2. Coarsely sized tetrahedral mesh of well-shaped elements of a) the antenna and the ground plane geometry and b) the complete computational model.

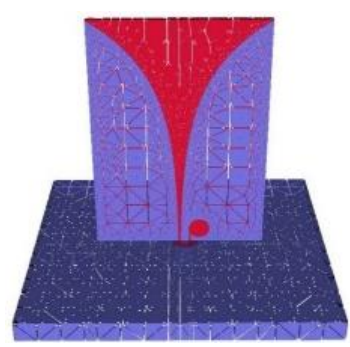

(a)

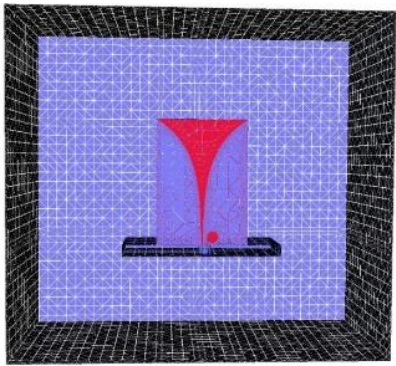

(b)
Fig. 3. a) Hybrid cubic-tetrahedral mesh of a) the antenna and the ground plane and b) the complete computational model.
All simulations in this section are run on 80 processor cores of a commodity cluster with a time step of $2 \mu \mathrm{m}$ and a threshold for forming cell clusters of $1 \mu \mathrm{m}$. (Physically, the modeler is managing the sensitivity to exact sampling placement and the maximum frequency of interest by this choice as shall be discussed further below). The geometry of the coaxial cable cross section is described using 50 piecewise linear segments as shown in Fig. 1b) which results in a very smooth cross-section, a decision that will be explored further below. The magnitude of the horizontally polarized electric field of the fundamental mode is shown in the inset of Fig. 4.

The normalized radiation intensity in the $\mathrm{H}$ - and $\mathrm{E}$ - plane is shown in Fig. 4a,b) respectively, together with the inset in each figure depicting how the patterns in each plane are defined. Fig. 4 shows that the coarsely sized tetrahedral mesh has a similar response to the $\lambda / 5$ hybrid mesh, neither resolving the far field patterns accurately due to inadequate sampling of the free space region. However, the field patterns do converge for the hybrid meshes with $\lambda / 10$ or better sampling, which corresponds to a cell size of $10 \mathrm{~mm}$ and smaller.

The results for this far field metric, suggest that a strategy of geometry resolution with good quality tetrahedra, bootstrapped by a maximum cell size chosen on the basis of the usual better than $\lambda / 10$ guideline, can provide useful results.

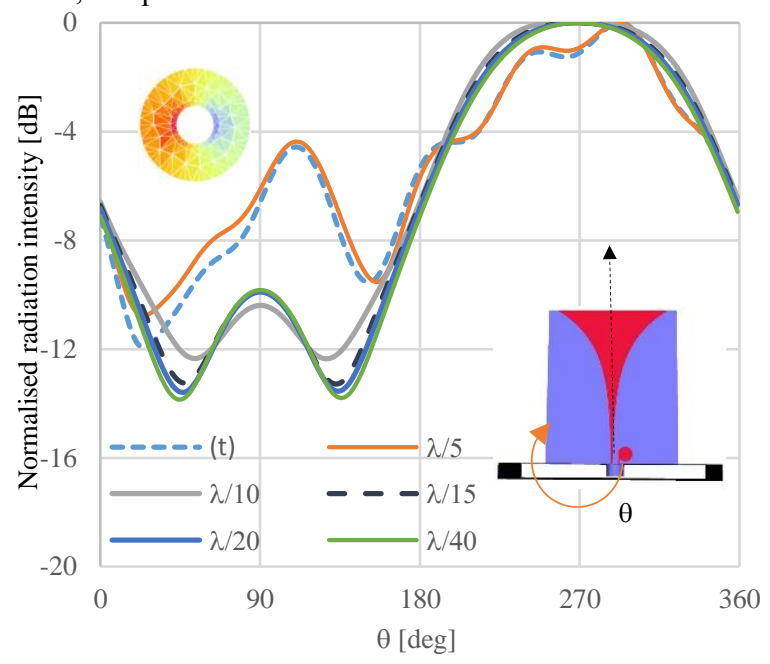

(a)

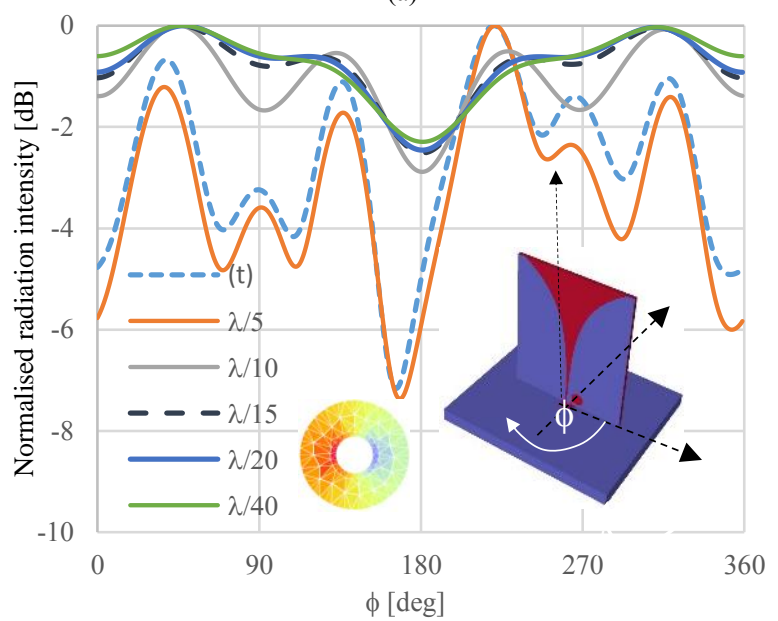

(b)

Fig. 4. Normalized radiation intensity in a) H-plane and b) E plane for different embedded cubic meshes of unit cell size $\lambda / 5, \lambda / 10, \lambda / .15, \lambda / 20$ and $\lambda / 40$ and (t) a coarsely sized good quality tetrahedral mesh. 
Fig. 6 shows the convergence of the return loss, $S_{11}$, obtained using the same range of meshes as above. It can be seen that coarsely sized tetrahedral mesh and hybrid meshes of cell size $\lambda / 10, \lambda / 15, \lambda / 20$ can resolve the depth of the return loss, $\mathrm{S}_{11}$, minimum, but the resonance is shifted to lower frequencies. Convergence is eventually obtained for the hybrid mesh of size $\lambda / 40$. The more stringent mesh requirement for return loss is clearly known to be attributable to the need to sufficiently resolve the near field of the antenna.

The computational runtime and peak memory are also given in Table 1. A number of factors explain why the run time in particular does not scale in a simple manner. First, the cubic cells are more computationally efficient than the tetrahedral cells as the code can be optimized around fewer (and shareable) characterizing parameters which can better utilize modern CPU caches. This explains the reduction in run time when comparing the pure tetrahedral case $(\mathrm{t})$ to the $\lambda / 10$ hybrid case. Straightforward use of finer grids has an underlying $4^{\text {th }}$ order scaling factor (3 spatial and 1 temporal). However, smaller cubes can approach more closely to the geometrical structure which also displaces slower tetrahedral cells. For reasonably large problems, the mesh often comprises a thin skin of tetrahedral, a few cells thick that provide a spatial transition from the geometrical scale of the surfaces to that of the cubic grid. Asymptotically, the relative number of tetrahedra and cuboids approaches a surface-tovolume relationship. This feature underpins the assertion of scalability for UTLM when used with hybrid meshes.

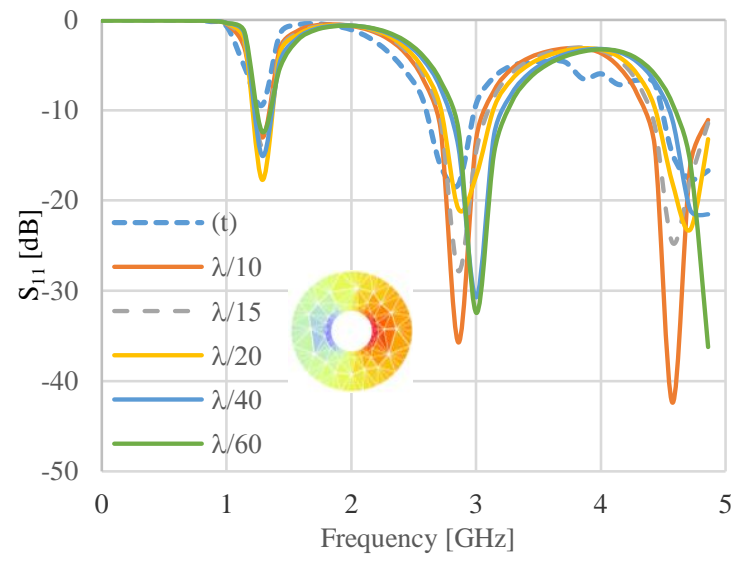

Fig. 5. $\mathrm{S}_{11}$ parameter for hybrid meshes with varying cubic mesh sizes.

\begin{tabular}{|c|l|l|}
\hline Mesh & Runtime (s) & Memory (MB) \\
\hline$t$ & 1972 & 288 \\
\hline$\lambda / 10$ & 973 & 225 \\
\hline$\lambda / 15$ & 1960 & 484 \\
\hline$\lambda / 20$ & 2362 & 529 \\
\hline$\lambda / 40$ & 2369 & 2033 \\
\hline$\lambda / 60$ & 3758 & 8290 \\
\hline
\end{tabular}

Table 1: Runtime and Memory Requirements for the results of Fig.5.

The second factor affecting runtime scaling is the computational nature of the cells clusters which was explored in depth in [34]. For coarse models, a few notably large cell clusters can form which disproportionally impact the run time, especially if one includes the pre-processing time (as done here) not just the time-stepping runtime. Typically, as the mesh is further resolved to practical, i.e. better than $\lambda / 10$ levels, these clusters often break up into smaller more efficient parts. However, it is clearly important that the modeler recognizes this phenomenon and understands how the definition of the CAD can impact upon it.

The results of Fig. 4 and Fig. 5 show that an accurate determination of $S_{11}$ requires a mesh four times smaller than that required for convergence of the field patterns and that refining the mesh will primarily require a notable increase in memory use.

Deploying a fine mesh throughout the problem space is prohibitive for large-scale simulations and we now explore the value of selectively refining the mesh locally around the antenna. Local mesh refinement is introduced by specifying a region in which a maximum target volume for the tetrahedral cells is defined. Our in-house meshing software permits refinement regions to be defined by means of mesh concentration points, lines or within fictitious cubic domains, as well as by proximity to selected surface features. Fig. 6 . provides a simple illustration; refinement within a non-physical box encompassing the structure within which a maximum target volume of $2 \mathrm{~mm}^{3}$ is prescribed and with a $\lambda / 10$ cubic background mesh. This can be regarded as the next level strategy, generally refine the near field region, but without focusing on particular elements of the geometry.

Fig. 7a,b) show the convergence of $S_{11}$ for the hybrid $\lambda / 10$ and $\lambda / 20$ meshes respectively, corresponding to cubic cell volumes of 1000 and $125 \mathrm{~mm}^{3}$. (A subtlety in comparing cell volumes is that a cube typically comprises 6 tetrahedra each of $\sim 1 / 6$ the cubic volume). On each plot, the different curves are for different target cell volumes for the near field refinement. To compare with the previous strategy, a reference result obtained with a fine $\lambda / 40$ hybrid mesh with no near field refinement is shown. As before, the inset of Fig. 7 also shows the magnitude of the horizontally polarized electric field profile of the coaxial mode which again remains the same due to the fact that cable discretization is still dominated by its smooth geometrical description.

In both Fig. 7a) and Fig. 7b) near field refinement improves the $S_{11}$ parameter now locating it at the correct resonant frequency. In fact, volume targets of below $2 \mathrm{~mm}^{3}$ further reduce $\mathrm{S}_{11}$, showing that Fig. 5 . had not fully converged in this regard. In comparison with the reference result obtained using the unrefined $\lambda / 40$ mesh, local refinement impacts the $S_{11}$ parameter by $7 \mathrm{~dB}$ or $4 \mathrm{~dB}$ for the hybrid $\lambda / 10$ and $\lambda / 20$ meshes respectively.

As a guideline, cell volumes below $2 \mathrm{~mm}^{3}$ are equivalent to tetrahedra with edge sizes of better than $\lambda / 40$ which further confirms the requirement that the near field of antenna typically need to be modeled with meshes of unit cell size of $\lambda / 40$.

Table 2 summarizes the computational resources, namely the simulation runtime and the peak memory usage. The simulation run time might initially be surprising. However, recalling that we do show total run time comprising both pre-processing and time stepping, a simple behavior is not to be expected. As stated above, the

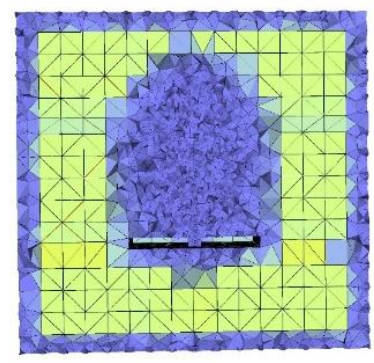

Fig. 6. Refined antenna near field mesh embedded within a $\lambda / 10$ cubic mesh. 
formation of cell clusters is a significant enabling feature and for these results the clustering threshold that controls this was kept constant which is physically justified as follows. The purpose of mesh refinement here is to better capture the rapidly varying near field behavior up to $5 \mathrm{GHz}$, knowing that this field behavior is intrinsically tied to geometrical features such as corners and the feed points. We are refining the mesh to achieve better spatial resolution of the quasistatic fields at the operating frequency, not to extend the fidelity of the model to higher frequencies by better capturing shorter wavelength behavior. The average spatial size of the clusters should be determined by the maximum frequency of interest and the role of mesh refinement is to give more resolution to the shape of the clusters. For example, seeking better resolution of the shape of a cluster of small cells forming around the end of a metallic dipole might be for the purpose of giving a more accurate estimator of the net capacitive lengthening effect at the fundamental operating frequency, not to extend the result to cover higher order resonances. Refining the mesh and reducing the clustering threshold would be the approach to obtaining a wider bandwidth.

Although the relationship between runtime and clustering parameters is still the subject of investigation, [34], the data in Table 2 does show that the gains in accuracy of Fig. 7 are not being bought by significant costs in resources, rather they support the underlying point of this work that intelligent targeting of resources is the critical issue. UTLM's clustering approach permits modelers an additional degree of freedom for this compared to many alternative methods.

In summary, in all cases of Fig. 7 significant memory savings are achieved by use of targeted mesh refinement within an adequately sampled, e.g. $\lambda / 10$, background mesh when compared to the unrefined $\lambda / 40$ mesh case without significantly affecting the overall runtime of the simulation.

Fig. 8a,b) compare the field patterns obtained using hybrid meshes of size $\lambda / 20$ and $\lambda / 10$ mesh, with and without near field mesh refinement. Fig. 8a) shows that local near field refinement within the $\lambda / 20$ mesh contributes very little to the accuracy of the radiation pattern in both the E- and H-plane as the field is already sufficiently well sampled for this purpose. The inset of Fig.8a) also shows that the radiating field patterns in a polar form are virtually indistinguishable. However, Fig. $8 b$ ) shows that with a coarser $\lambda / 10$ hybrid mesh, near field refinement does change the radiation pattern. Fig. 8b) also compares the field patterns with the unrefined hybrid $\lambda / 20$ mesh and shows that near field refinement somewhat improves the radiation pattern in the sidelobes of the H-plane compared to the $\lambda / 20$ mesh and gives a better estimate for the radiation pattern. However, when comparing the memory requirements, the hybrid $\lambda / 20$ mesh requires $529 \mathrm{MB}$ (Fig. 5), and locally refined $\lambda / 10$ mesh with a target volume of $2 \mathrm{~mm}^{3}$ requires $412 \mathrm{MB}$ (Fig. 5) - a saving of $23 \%$.

It is concluded from this section that, in cases of large-scale scenarios and limited memory resources sampling at $\lambda / 10$, but more conservatively $\lambda / 20$, in conjunction with simplistically defined near field refinement can offer a valid compromise for good initial prediction of both $\mathrm{S}_{11}$ and the radiation pattern.

The next level of sophistication consider is to target particular features of the near field geometry to which the results are expected to be particularly sensitive. It is commented that although this might seem an obvious step, for complicated configurations containing many such sub-structures, antennas, wires, fasteners etc, the need for the modeler to manually intervene in the mesh targeting becomes less attractive and there is definitely a role for the simplistic near field box approach just described. Nevertheless, it is valuable to explore the further benefits that can be obtained by a more focused mesh refinement.

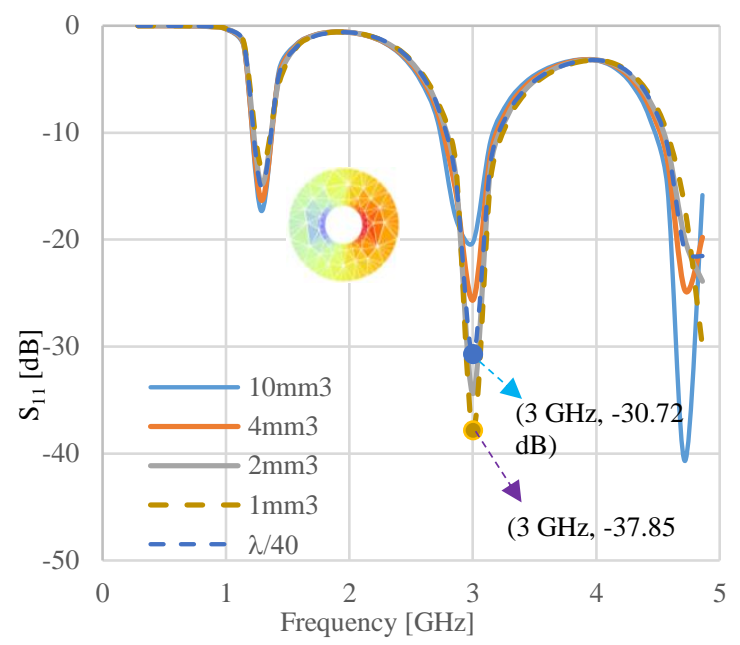

(a)

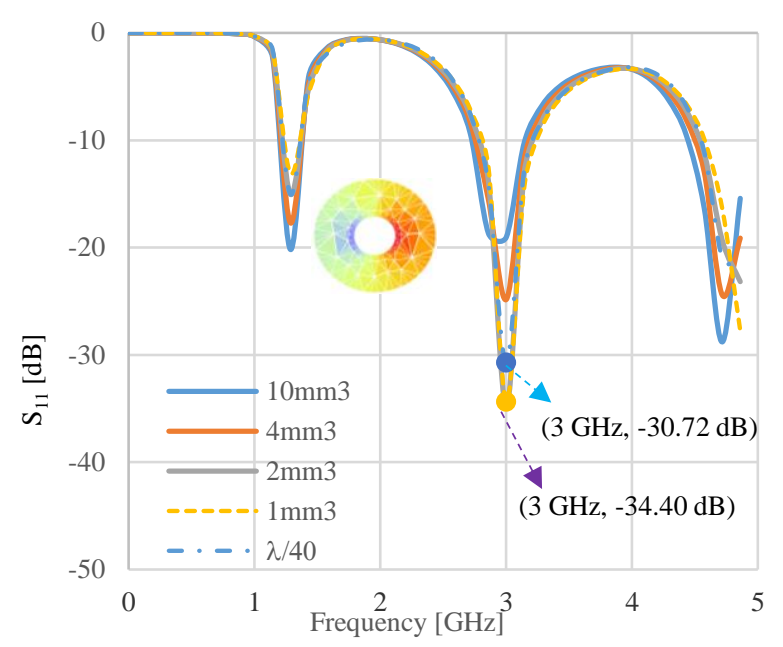

(b)

Fig. 7. Comparison of $S_{11}$ parameter for different near field mesh refinements, (target volume cell of $1-10 \mathrm{~mm}^{3}$ ) with a) a hybrid $\lambda / 10$ background cubic mesh and b) a hybrid $\lambda / 20$ background cubic mesh.

\begin{tabular}{|c|l|l|l|}
\hline Background Mesh & $\begin{array}{l}\text { Near field } \\
\text { volume target, } \mathrm{mm}^{3}\end{array}$ & Runtime (s) & Memory (MB) \\
\hline$\lambda / 40$ & Unrefined & 2369 & 2033 \\
\hline$\lambda / 10$ & 10 & 2477 & 344 \\
\hline$\lambda / 10$ & 4 & 2479 & 369 \\
\hline$\lambda / 10$ & 2 & 2482 & 412 \\
\hline$\lambda / 10$ & 1 & 2487 & 493 \\
\hline$\lambda / 20$ & 10 & 2807 & 637 \\
\hline$\lambda / 20$ & 4 & 2965 & 662 \\
\hline$\lambda / 20$ & 2 & 2963 & 703 \\
\hline$\lambda / 20$ & 1 & 2876 & 805 \\
\hline
\end{tabular}

Table 2: Runtime and Memory Requirements for the results of Fig. 5. 


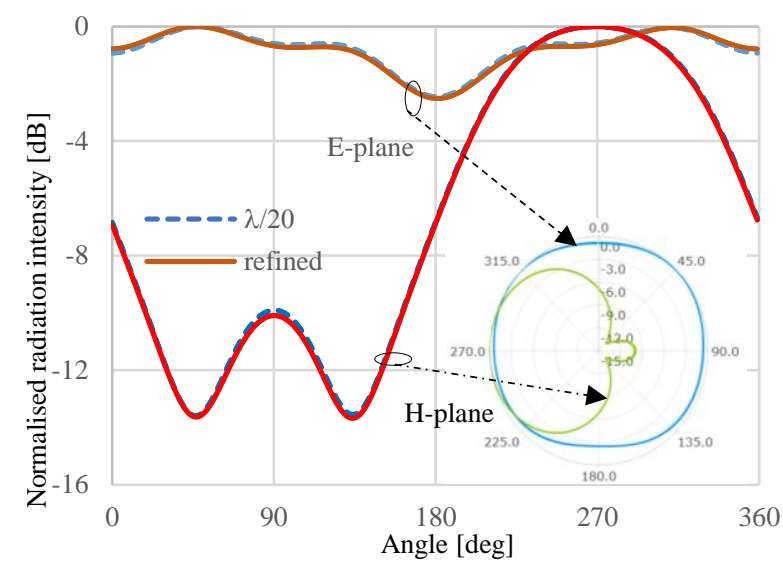

(a)

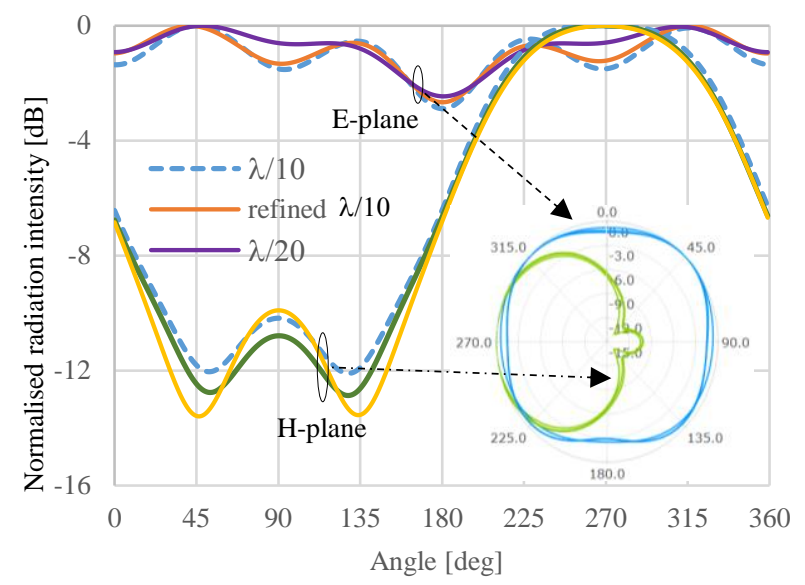

(b)

Fig. 8. Normalized radiation intensity in the E- and $\mathrm{H}$ - plane for a) the $\lambda / 20$ hybrid mesh with refinement (solid line) and without refinement (dashed line) and b) the $\lambda / 10$ hybrid mesh, with refinement (solid line), without refinement (dashed line) compared with hybrid $\lambda / 20$ mesh (solid line). The inset in figures gives the normalized field patterns in the polar form

All of the preceding results were obtained with the circumference of the coaxial cable geometry described by 50 piecewise linear segments. The resulting smooth curvature combined with the requirement for a good quality mesh results in a rather fine mesh discretization of the coaxial cable cross-section albeit at the expense of overall mesh size and memory requirements. In order to investigate if the geometrical description of the coaxial cable can be relaxed without unduly affecting the accuracy of the simulation, the circumference of the cable has also been modeled with 4, 8, 20,30, 40 and 50 segments. For this investigation the $\lambda / 20$ hybrid mesh is used with a near field target cell volume of $2 \mathrm{~mm}^{3}$ selected. The magnitude of the horizontally polarized electric field for different cable cross sections is shown in the inset of the Fig. 9a) and it can be seen how the geometrical resolution impacts the field resolution of the fundamental mode solution which is exciting the antenna. Fig. 9a) shows that even when the coaxial cable is described very crudely with only 4 segments the simulation can still accurately predict the resonant frequencies of $S_{11}$ but there is an error in the depth of the resonance of more than $15 \mathrm{~dB}$ at $3 \mathrm{GHz}$ when compared to the results using cross sections of 30 or 50 segments.

One point to observe is that the run time in Table 3 is actually notably higher for the crudest cable description. Again, this is attributable to the formation of clusters, in this case around the 90- degree corner of the cable. As the resolution of the cable is most likely set by the CAD data before meshing, this reinforces the argument that the modeler must be aware of the impact on computational effort of many more issues than just the simulation methodology.

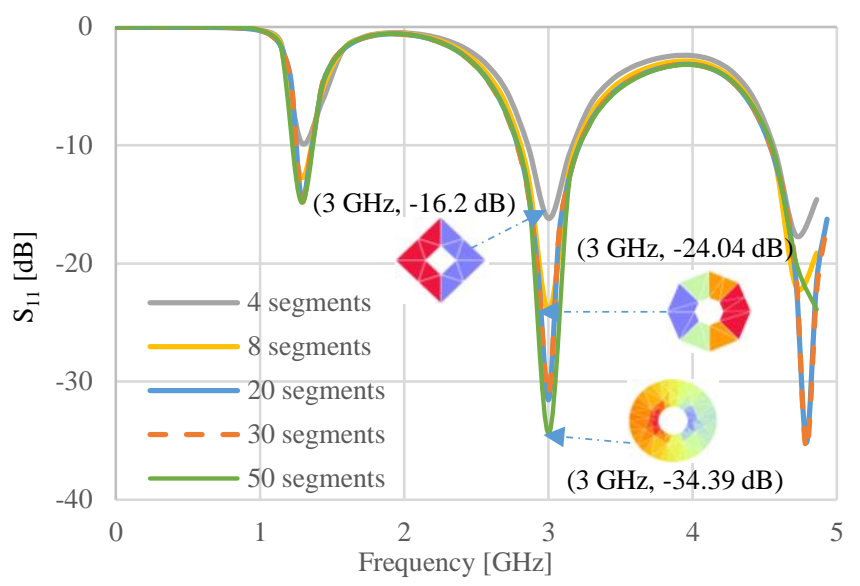

(a)

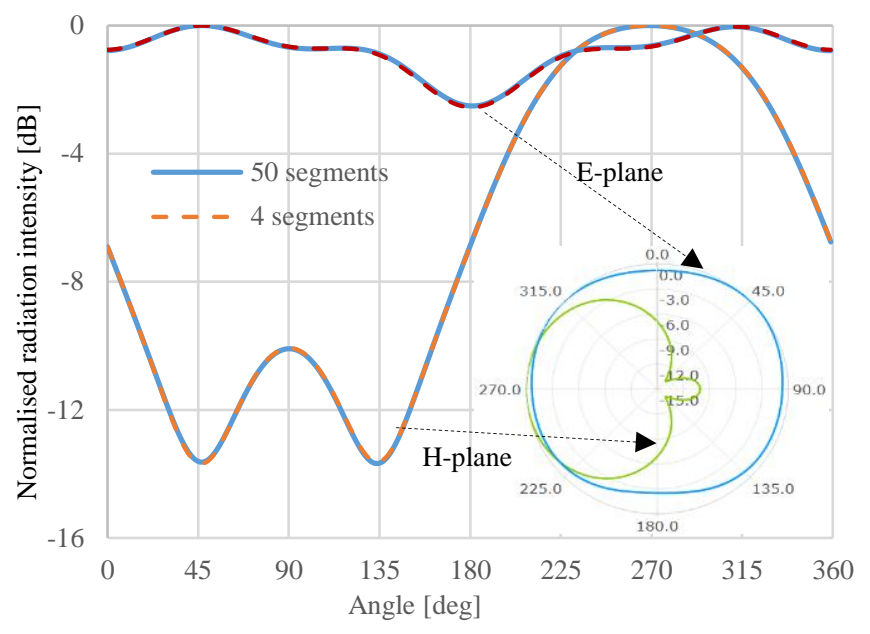

(b)

Fig. 9. a) Comparison of $S_{11}$ and b) Normalized radiation fields obtained using coaxial cable described with 4 and 50 circumferential segments.

\begin{tabular}{|c|l|l|l|l|}
\hline $\begin{array}{l}\text { Background } \\
\text { Mesh }\end{array}$ & $\begin{array}{l}\text { Near field } \\
\text { volume } \\
\text { target, } \mathrm{mm}^{3}\end{array}$ & $\begin{array}{l}\text { Coaxial } \\
\text { Cable } \\
\text { resolution, } \\
\text { segments }\end{array}$ & Runtime (s) & $\begin{array}{l}\text { Memory } \\
\text { (MB) }\end{array}$ \\
\hline$\lambda / 20$ & 2 & 4 & 2048 & 575 \\
\hline$\lambda / 20$ & 2 & 8 & 433 & 573 \\
\hline$\lambda / 20$ & 2 & 20 & 378 & 384 \\
\hline$\lambda / 20$ & 2 & 30 & 695 & 587 \\
\hline$\lambda / 20$ & 2 & 50 & 2361 & 703 \\
\hline
\end{tabular}

Table 3. Runtime and Memory Requirements for the results of Fig.9.

Fig. 9b) compares the radiating fields in the E- and $\mathrm{H}$ - plane obtained when the antenna is excited using a crude coaxial cable geometry described by 4 segments and a fine geometry described by 50 segments and shows that the geometrical description of the cable does not affect the far field pattern, the results being indistinguishable in both forms of the plots. This is an encouraging result as it shows that valuable computational savings can be made when analysis of far 
field patterns is the principle objective of the simulation. For example, a $20 \%$ saving in the memory and a $30 \%$ saving in the runtime is obtained by describing a coaxial cable crudely with 4 segments compared to a fine geometrical description of 50 segments without affecting the far field pattern.

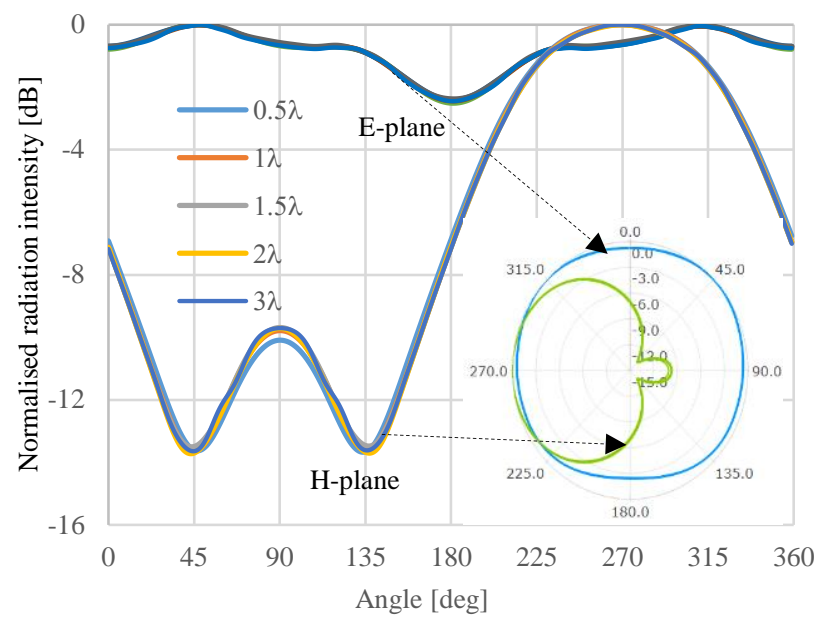

(a)

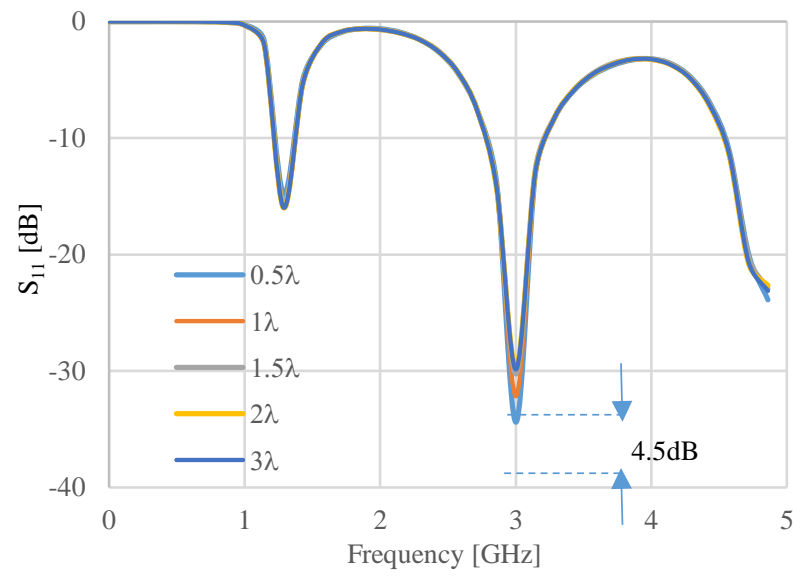

(b)

Fig. 10. a) Normalized radiation intensity in the E-plane and H-plane and b) $\mathrm{S}_{11}$ parameter for different sizes of the computational box.

\begin{tabular}{|c|l|l|l|l|}
\hline $\begin{array}{l}\text { Background } \\
\text { Mesh }\end{array}$ & $\begin{array}{l}\text { Near field } \\
\text { volume } \\
\text { target, } \mathrm{mm}^{3}\end{array}$ & $\begin{array}{l}\text { Computational } \\
\text { box size }\end{array}$ & $\begin{array}{l}\text { Runtime } \\
(\mathrm{s})\end{array}$ & $\begin{array}{l}\text { Memory } \\
(\mathrm{MB})\end{array}$ \\
\hline$\lambda / 20$ & 2 & $0.5 \lambda$ & 2963 & 662 \\
\hline$\lambda / 20$ & 2 & $1.0 \lambda$ & 2550 & 1328 \\
\hline$\lambda / 20$ & 2 & $1.5 \lambda$ & 3268 & 2836 \\
\hline$\lambda / 20$ & 2 & $2.0 \lambda$ & 3285 & 5287 \\
\hline$\lambda / 20$ & 2 & $3.0 \lambda$ & 9189 & 14459 \\
\hline
\end{tabular}

Table 4: Runtime and Memory Requirements for the results of Fig. 10.

The impact of the size of the computational box on the antenna radiation intensity and $S_{11}$ parameter is investigated in Fig. 10. The computational problem space is terminated at $0.5 \lambda, \lambda, 1.5 \lambda, 2 \lambda$ and $3 \lambda$ away from the antenna-ground plane geometry. In all cases a hybrid $\lambda / 20$ mesh, further refined in the antenna's near field region using a cell volume target of $2 \mathrm{~mm}^{3}$ is used and the coaxial cable is described by 50 segments. Fig. 10a) shows the normalized radiation intensity in the E- and H-plane and Fig. 10b) shows $\mathrm{S}_{11}$. It can be seen that in all cases the size of the computational box does not substantially affect the results. However, a closer inspection shows that truncating the computational box more than $1.5 \lambda$ away from the antenna gives well converged results for both radiation pattern and $\mathrm{S}_{11}$. Fig. $10 \mathrm{~b}$ ) also shows that truncating the computational box too close to the antenna, i.e. at $0.5 \lambda$ from the antenna, gives rise to an error of $4.5 \mathrm{~dB}$ in the value of $\mathrm{S}_{11}$ at $3 \mathrm{GHz}$, compared to the larger box terminated at $1.5 \lambda$. Details of the computational requirements for the different computational box sizes are given in Table 4 indicating that significant savings can be made by optimizing the size of computational box. Here, the run times with the smaller boxes are dominated by the effort for the tetrahedral cells, but with larger boxes this changes to domination by the cubic mesh and the scaling becomes asymptotically more predictable.

\section{B. Vivaldi Antenna on a Large Ground Plane}

The previous section explored the accuracy of antenna modeling on a small ground plane of the size $0.7 \lambda \times 0.6 \lambda$. This is an important step as it fully characterizes the antenna's performance but it does not take into account the realistic setting of the antenna. The installation of antenna is an important aspect to consider and in this section the changes in antenna performance due to installation are investigated by considering platforms that span sizes ranging from the near to the far field of antenna.

Fig. 11 and Fig. 12 explore the effect of the antenna installation on a square platform having a side length of $\lambda, 6 \lambda$ and $10 \lambda$, where $\lambda$ is the operating frequency of the antenna. In all cases a $\lambda / 20$ hybrid mesh is used with target cell volume of $2 \mathrm{~mm}^{3}$ in the antenna's near field region and with the computational box truncated at a distance of $1.5 \lambda$ away from the structure. All simulations are run with a time step corresponding to using of a $2 \mu \mathrm{m}$ cubic cell and a cell clustering threshold of $1 \mu \mathrm{m}$. Fig. 11 compares $S_{11}$ for different platform sizes and shows that the size of the platform does not affect the position of resonant frequencies of the antenna but mainly affects the depth of the minima of $\mathrm{S}_{11}$, which changes by $12.95 \mathrm{~dB}$ at $3 \mathrm{GHz}$ and by $11 \mathrm{~dB}$ at $1.2 \mathrm{GHz}$ with platform sizes of $0.7 \lambda$ and $10 \lambda$. This is a significant change in $S_{11}$ prediction and contradicts the result of [19] which showed that the large platform of similar electrical size $(8 \lambda \times 6 \lambda)$ had negligible effect on $\mathrm{S}_{11}$. Table 5 also compares computational resources for each installation case showing significant increase in computational resources as the problem size is increased. Again, the scaling behavior of the runtimes is strongly affected by cluster formation.

Fig. 12a,b) shows the normalized radiation pattern in the E- and $\mathrm{H}-$ planes respectively. Fig. 12a) shows that as the platform size is increased, the radiation pattern becomes more directional in the Eplane. In the H-plane, the radiation intensity in both the main beam and the side-lobes is decreased, the nulls in the radiation pattern are shifted and the number of side lobes is increased, as shown in Fig. 12b).

Fig. 11 and Fig. 12 confirm that the impact of installation on antenna performance is practically significant and modeling antennas in their practical environment must be the goal. All the results in Fig. 11 and Fig. 12 were obtained with a hybrid mesh of unit cell size $\lambda / 20$, however use of such a fine mesh becomes computationally impractical for ever larger electrically large platforms. Therefore, there is a strong motivation to investigate the use of coarser background meshes. More sophisticated mesh grading strategies could exploit the fact that the surface current density on the platform decreases significantly with distance from antenna. However, this places yet more requirements on the modeler and the mesh generation software and in the context of full aircraft models with many such installed components, will 
demand a substantial human effort. Therefore here, we simply explore whether use of a hybrid mesh with a cruder cubic background grid produces sufficiently useful results. For reference, it is noted that a minimum sampling of $\lambda / 20$ using a structured sugar cube FDTD grid is reported in [2] and triangles with edge size of $\lambda / 8$ and $\lambda / 10$ are reported in [1] with 2 times denser mesh around the antenna.

Fig. 13 compares the effect of the discretization on the accuracy of the radiation pattern for an electrically large, $10 \lambda$ platform. Specifically, Fig. 13a,b) show the normalized radiation pattern in the $\mathrm{E}$ - and $\mathrm{H}$ - planes using a hybrid mesh sampling of $\lambda / 10$ and $\lambda / 8$ for a large platform, respectively, in each case comparing results with those obtained using a hybrid mesh of $\lambda / 20$ for both the large, $10 \lambda$, and the small $0.7 \lambda$ platform.

Fig. 13a) shows that installation effects play a dominant role in antenna field patterns and that the results from the hybrid $\lambda / 10$ and $\lambda / 20$ meshes are very similar in the main radiation pattern and location of the nulls. However, the most significant point is that the difference in patterns when installed on the small and large platforms is substantially greater than the errors caused by using the cruder $\lambda / 10$ mesh. This is a significant result as it illustrates that highly accurate models of isolated antennas, for example obtained using very fine meshes, are of limited value in assessing installed performance. This point is further reinforced by Fig. 13b), when even the use of a mesh for the installed case that would break the conventional sample better than $\lambda / 10$ rule gives better results than a finely meshed isolated case.

Table 6 compares computational resources for each case of Fig. 13 and showing that significant savings can be made if cruder hybrid meshes are used when modeling antennas on large platforms without significant loss of accuracy.

A further significant point is that the non-uniform meshing strategy has provided accurate results using a background sampling density of $\lambda / 10$ and $\lambda / 8$ which is drastically lower than the FDTD method that required a minimum background sampling of $\lambda / 20$ [2] and is comparable to those of the frequency domain multi-level fast multiple method (MLFMM) [1].

As a further example of where designer understanding of the physical mechanisms can be exploited, Fig. 14 explores whether further computational savings can be made by truncating the computational box just below the ground plane for platforms of size $10 \lambda$ and $6 \lambda$ where little field is expected to exist. In all cases a $\lambda / 10$ hybrid mesh with target cell refinement of $2 \mathrm{~mm}^{3}$ in the antenna near field is used. For the $10 \lambda$ platform both full space and half space box simulations predict very similar results as shown in Fig. 14a) whereas for the $6 \lambda$ platform small differences appear in the amplitudes of minor side-lobes but the main features in the pattern are preserved. In both cases significant computational savings are made by reducing the computational box as outlined in Table 7, with a $34 \%$ saving in the memory and a $46 \%$ saving in the runtime for the case of the $10 \lambda$ platform.

\begin{tabular}{|c|l|l|l|l|}
\hline $\begin{array}{l}\text { Background } \\
\text { Mesh }\end{array}$ & $\begin{array}{l}\text { Near field } \\
\text { volume } \\
\text { target, } \mathrm{mm}^{3}\end{array}$ & $\begin{array}{l}\text { Platform } \\
\text { size }\end{array}$ & Runtime (s) & $\begin{array}{l}\text { Memory } \\
(\mathrm{MB})\end{array}$ \\
\hline$\lambda / 20$ & 2 & $0.7 \lambda$ & 3336 & 2971 \\
\hline$\lambda / 20$ & 2 & $1.0 \lambda$ & 3295 & 3382 \\
\hline$\lambda / 20$ & 2 & $6.0 \lambda$ & 9756 & 14510 \\
\hline$\lambda / 20$ & 2 & $10.0 \lambda$ & 13470 & 20010 \\
\hline
\end{tabular}

Table 5: Runtime and Memory Requirements for the results of Fig. 11.

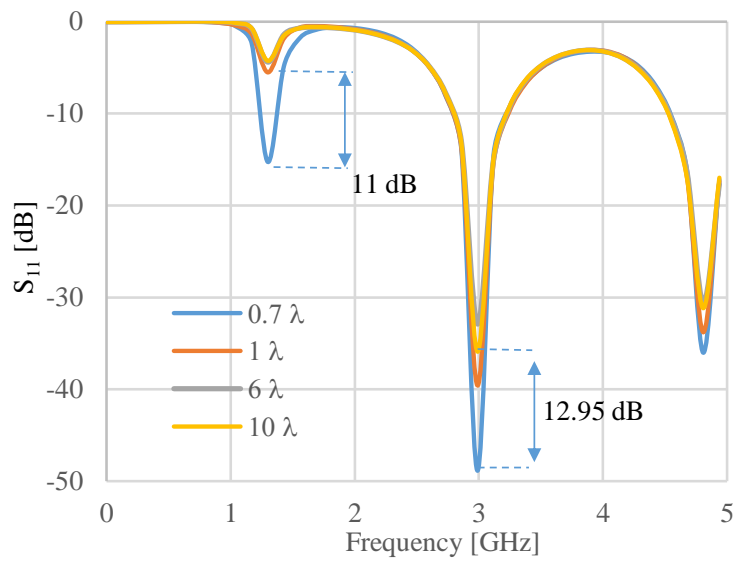

Fig. 11. Comparison of $\mathrm{S}_{11}$ parameter, computational resources and the largest cluster size for the antenna installed on a square platform with side length of $0.7 \lambda, 1 \lambda, 6 \lambda$ and $10 \lambda$.

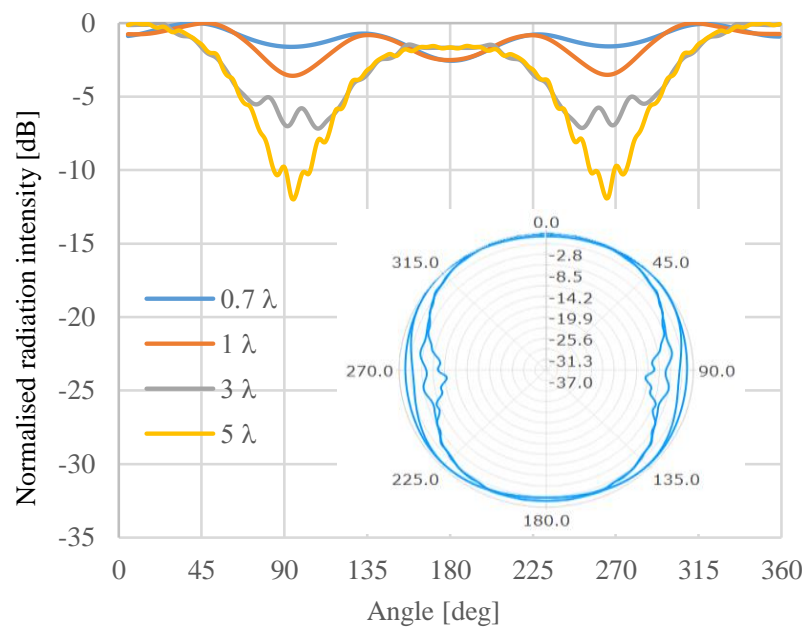

(a)

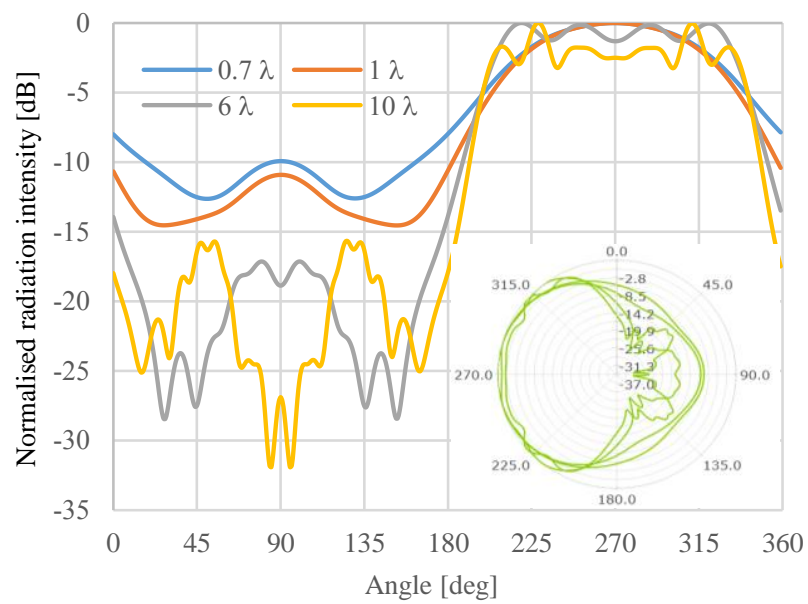

(b)

Fig.12. Normalized radiation intensity in a) H-plane and b) E-plane for square platforms with side length of $0.7 \lambda, 1 \lambda, 6 \lambda$ and $10 \lambda$. The inset of the figure shows the results in polar form. 
0

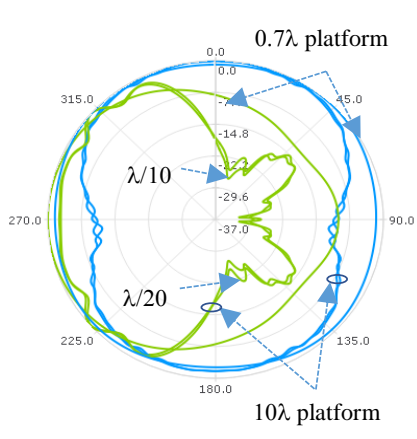

(a)

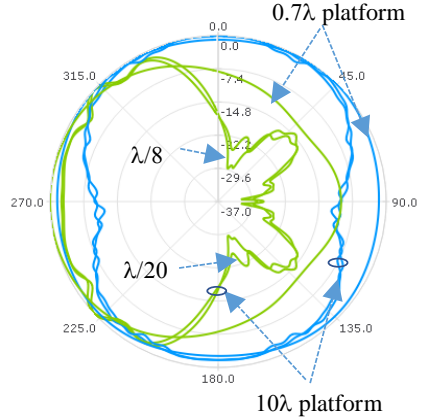

b)
Fig. 13 Comparison of the radiation fields obtained using hybrid meshes of a) $\lambda / 10$ and $\lambda / 20$ cell size for electrically large platform of $10 \lambda$ with that of a hybrid mesh of $\lambda / 20$ for the antenna installed on a small $0.7 \lambda$ platform; b) $\lambda / 8$ and $\lambda / 20$ cell size for electrically large platform of $10 \lambda$, with that of a hybrid mesh of $\lambda / 20$ for the antenna installed on a small $0.7 \lambda$ platform.

\begin{tabular}{|c|l|l|l|l|}
\hline $\begin{array}{l}\text { Background } \\
\text { Mesh }\end{array}$ & $\begin{array}{l}\text { Near field } \\
\text { volume } \\
\text { target, } \mathrm{mm}^{3}\end{array}$ & $\begin{array}{l}\text { Platform } \\
\text { size }\end{array}$ & Runtime (s) & $\begin{array}{l}\text { Memory } \\
\text { (MB) }\end{array}$ \\
\hline$\lambda / 20$ & 2 & $0.7 \lambda$ & 3336 & 2971 \\
\hline$\lambda / 8$ & 2 & $10.0 \lambda$ & 3005 & 2592 \\
\hline$\lambda / 10$ & 2 & $10.0 \lambda$ & 3663 & 4436 \\
\hline$\lambda / 20$ & 2 & $10.0 \lambda$ & 7334 & 11565 \\
\hline
\end{tabular}

Table 6: Runtime and Memory Requirements for the results of Fig. 13.

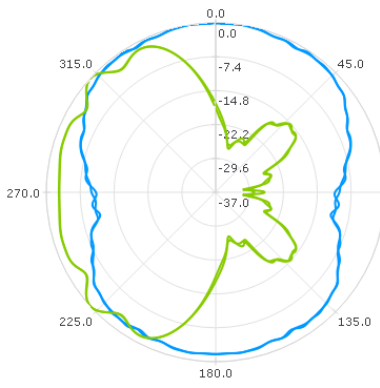

(a)

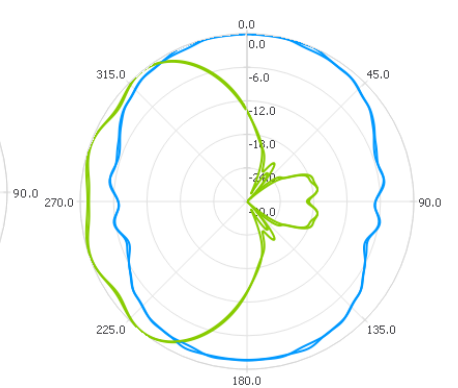

(b)
Fig. 14. Normalized radiation intensity for the antenna on the a) $10 \lambda$ and b) $6 \lambda$ platforms with reduced and full computational boxes.

\begin{tabular}{|c|l|l|l|l|}
\hline $\begin{array}{l}\text { Background } \\
\text { Mesh }\end{array}$ & $\begin{array}{l}\text { Truncated } \\
\text { Computational } \\
\text { box }\end{array}$ & $\begin{array}{l}\text { Platform } \\
\text { size }\end{array}$ & $\begin{array}{l}\text { Runtime } \\
(\mathrm{s})\end{array}$ & $\begin{array}{l}\text { Memory } \\
\text { (MB) }\end{array}$ \\
\hline$\lambda / 8$ & No & $6 \lambda$ & 3663 & 4436 \\
\hline$\lambda / 10$ & Yes & $6 \lambda$ & 1982 & 2946 \\
\hline$\lambda / 10$ & No & $10.0 \lambda$ & 2914 & 1647 \\
\hline$\lambda / 20$ & Yes & $10.0 \lambda$ & 894 & 2458 \\
\hline
\end{tabular}

Table 7: Runtime and Memory Requirements for the results of Fig. 14.

\section{VIVALDI ANTENNA INTEGRATED IN AN AIRCRAFT WING}

This section explores how the installation of an antenna in the leading edge of an aircraft wing affects its performance. Besides being more realistic, the curved nature of this platform further motivates the adoption of an unstructured mesh approach such as UTLM.
Below, we investigate the impact of the presence of the platform, the antenna's orientation within it and the introduction of a radome cover. The antenna is the same broadband Vivaldi antenna of sections 3.1 and 3.2 and the perfectly conducting wing is derived from an NACA 0010 airfoil with chord length $1.83 \mathrm{~m}$, maximum thickness over chord of $0.18 \mathrm{~m}$ and a span of $3.35 \mathrm{~m}$. Fig. 15 shows the antenna in situ where $w_{l}$ and $w_{2}$, are dimensions of the flat platform on which antenna is mounted, $t$ is the thickness of the platform, $d$ is the depth at which the platform is positioned from the leading edge of the wing and $s$ is the overall length of the wing section. All simulations are performed using a $10 \mathrm{~mm}$ mesh refined in the antenna near field with the target cell volume of $2 \mathrm{~mm}^{3}$.

The choice to retain just the leading edge, rather than the full wing is typical of the role of modeler experience and during an iterative design process. Whilst installation in the full aircraft model might be the eventual goal, focusing attention in the first instance on the most significant installation effects provides a pragmatic compromise with computational effort. Therefore, as in the previous section, we also define the intermediate case where the antenna is just placed on a simple flat conducting plate of size $w_{l}$ and $w_{2}$ for comparison.

Similarly, space precludes repeating the full investigation of section III and the meshing choices for the selected simulations presented here will be based upon the conclusions of that work. Again, this is where the modeler's experience must be drawn upon. Physically, the rapidly varying quasi-static near field antenna fields within the wing context are still primarily interactions with the plate it is mounted upon and thus the analysis of section III is a sound basis for selecting parameters. The remaining mechanisms for installation interactions are with the vertical ribs of the wing and the diffractive phenomena at the edges. Comparing the wavelength to the rib spacing and given the expected field strengths, these do not give cause for concern for the parameter choice. Of course, good practice for any simulation motivates confirmation of this argument, but space precludes it here.

Fig. 16 compares the normalized radiation patterns in the E- and $\mathrm{H}-$ plane when the antenna is installed in the wing without the radome cover to those when installed on the simple flat plate. The model parameters are $w_{l}=0.35 \mathrm{~m}, w_{2}=0.28 \mathrm{~m}, t=30 \mathrm{~mm}, d=0.2 \mathrm{~m}$ and $s=2.15$ $\mathrm{m}$. It is immediately clear that installation in the wing significantly changes the results. In the H-plane the sidelobe level is lower by 10 $\mathrm{dB}$, the radiation nulls are shifted and the intensity in the main beam is slightly reduced for the case of antenna installed in the wing. In the E-plane the pattern is more directional and possess more nulls compared to the case of antenna on a flat platform.

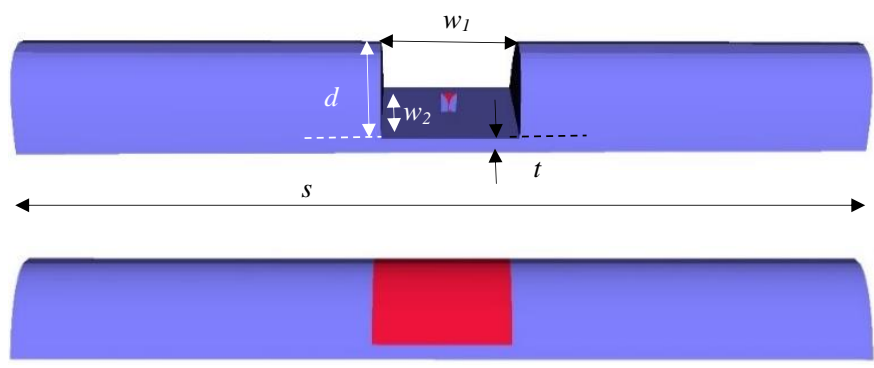

Fig. 15. Vivaldi antenna installed in the leading edge of the wing facing forward, with and without a radome cover in place.

Fig. 17 considers an alternative orientation of the antenna, rotating it by 90 degrees so that it faces the wing ribs and compares the field patterns for the two orientations. As expected, the results confirm that the performance of the antenna facing the wing is significantly 
degraded due to reflections from the wing structure, especially in the H-plane.

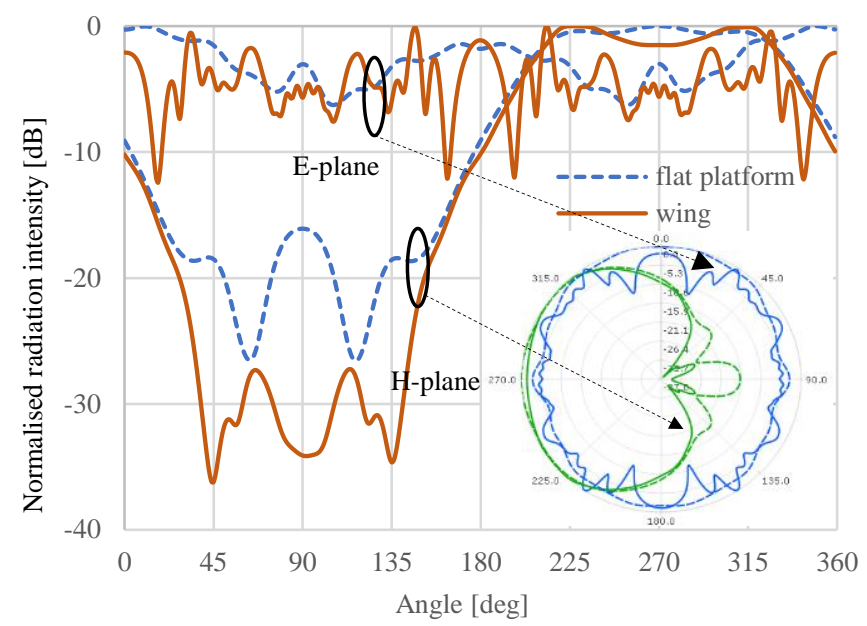

Fig. 16. Comparison of normalized radiation intensity between antenna installed in the wing and installed on a flat platform.

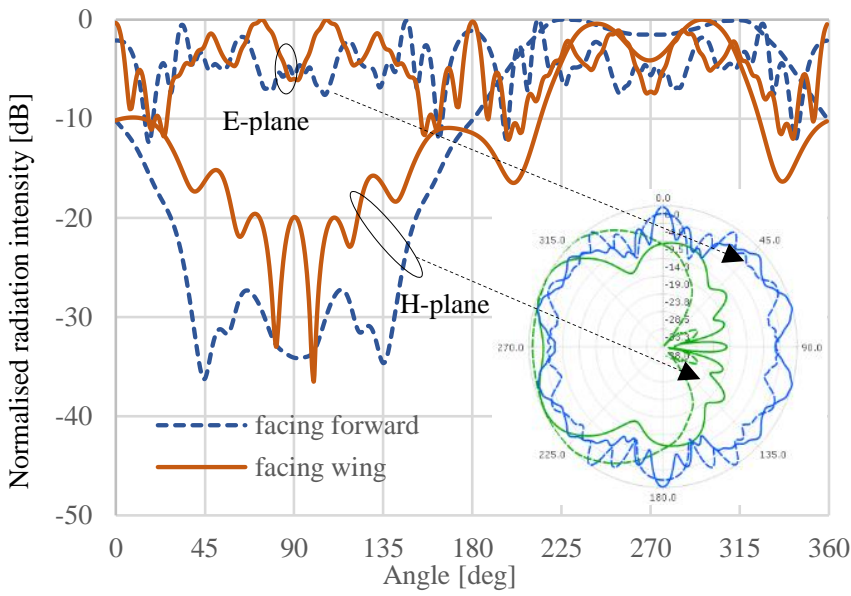

Fig. 17. Comparison of normalized radiation intensity for antenna installed in the wing facing forward and facing one of the wing ribs.

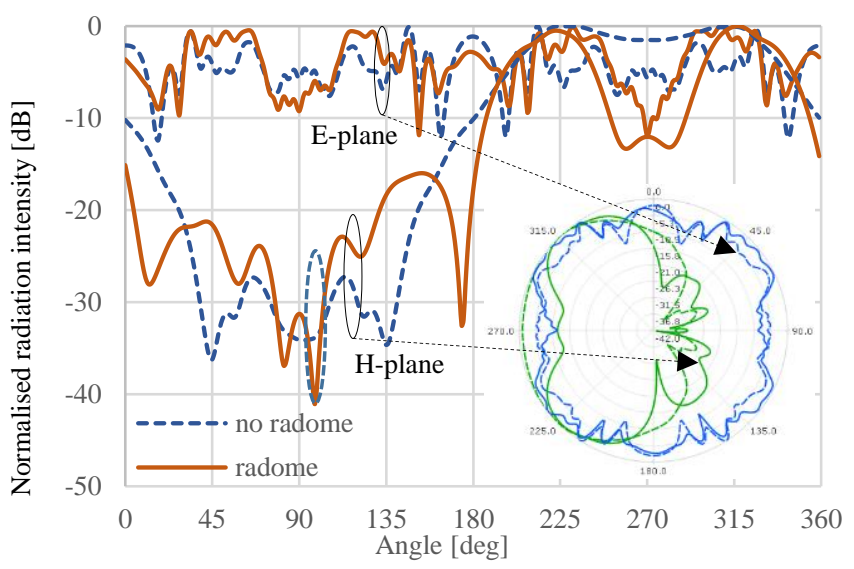

Fig. 18. Comparison of normalized radiation intensity for antenna installed in the wing as in a) with and without the radome cover.

The impact of the radome cover on antenna performance is now considered. The cover is a half-wavelength dielectric layer of permittivity $\varepsilon_{\mathrm{r}}=4.2$ and thickness of $24.4 \mathrm{~mm}$ designed to operate at 3 GHz. Fig. 18 shows that the presence of the radome significantly degrades the antenna performance by severely flattening the gain in the main beam in the H-plane and increases unwanted radiation in the sidelobes, whilst the antenna is more directive in the E-plane, with a prominent null at 270 degrees.

Finally, Fig. 19 shows the $\mathrm{S}_{11}$ parameter for the antenna in the wing with and without the radome cover. The results show that consideration of the realistic setting of the wing changes the magnitude of $\mathrm{S}_{11}$ at $3 \mathrm{GHz}$ by $16 \mathrm{~dB}$ compared to the flat platform case. $\mathrm{S}_{11}$ is additionally changed by the presence of the radome cover with noticeable ripples in the response that are due to the antennas interaction with it.

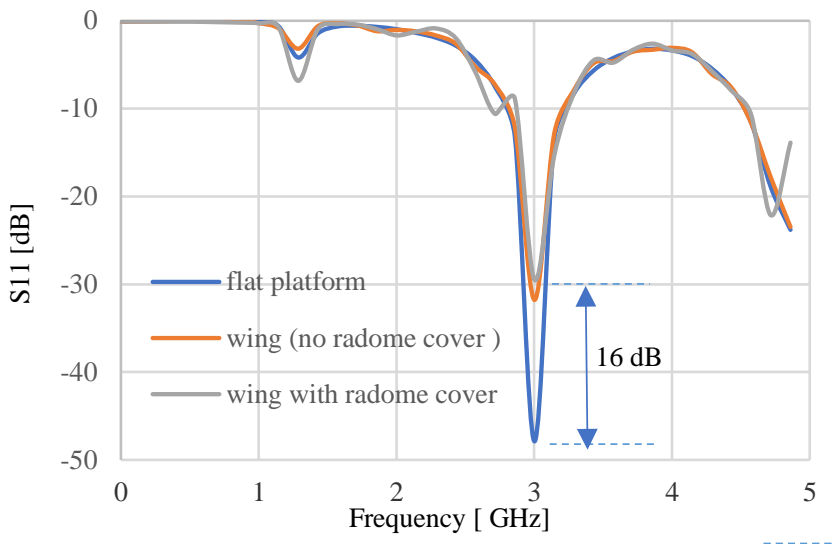

Fig. 19. Comparison $\mathrm{S}_{11}$ for the antenna installed on the flat platform, in the wing with no radome cover and in the wing with the radome cover.

In summary, these results confirm that the performance of the antenna installed in the aircraft wing will strongly depend upon the details of its integration within the wing, i.e. the size of the mounting plate, the depth of the platform from the leading edge and the presence of the radome cover. This paper does not have the space to explore optimization, rather sought to demonstrate how antenna behavior must be assessed in context rather than in isolation.

\section{CONCLUSION}

Antenna performance is significantly changed when installed in realistic environments, being affected by both near and far field interactions. As many applications ranging from aerospace through to the domestic are equipped with ever more antennas and other sensors, there is a strong demand to provide predictive simulations of complete configurations, rather than to rely on uncoupled models.

Full wave numerical methods are powerful candidates to provide this capability but are computationally challenging and must be deployed with careful focusing of their resources. Unstructured mesh approaches are adept at reducing sampling densities and time stepping algorithms are capable of accounting for both broadband signals and a diverse range of exotic materials.

For this reason, this paper has explored the issues of installed antenna performance using the UTLM method for the first time. The method's accuracy was already established, but its applicability to this scenario has now been confirmed by its use for a sequence of typical examples. The objective here has not been to present highly accurate results for particular antennas, rather to investigate whether relatively crude models are nevertheless valuable. Indeed, it has been shown that cruder models accounting for the installation are actually more useful that highly accurate models in isolation. 
A theme has been developed of understanding which features of a model impact most on the value of the results. Building upon simple concepts, a sequence of issues has been investigated demonstrating the tradeoffs between accuracy and computational effort. An awareness of realistic CAD data and mesh generation has informed this investigation and it is reiterated that showing that ideas such as near field refinement defined by simple boxes produce valuable gains is useful as trying to adopt more sophisticated schemes in complex configurations is likely to prove difficult to automate or demand high levels of human intervention.

In particular, the paper has assessed how realistic installation of an antenna affects both the near field return loss and far field radiation pattern of the antenna. Three different environment scenarios are considered: antenna in isolation, i.e. on an electrically small platform, on an electrically large platform and installed in the leading edge of an aircraft wing.

Specific results considered the required meshing for modeling antenna in isolation, distinguishing between the background cubic grid, refinement of the near field region, the impact of the feedline resolution and the size of the computational box. It is first clear that the use of hybrid tetrahedral-cubic meshes is a major asset in reducing runtime and memory. Further, substantial computational savings can be made by locally refining the mesh around antenna near field for accurate estimations of $S_{11}$. The paper shows that using a crude coaxial cable geometry does not compromise the accuracy of the far field pattern but that for a better estimation of the $S_{11}$ parameter at least 30 piecewise sections in the cable cross section should be considered. Truncating the computational box at least $1.5 \lambda$ away from the antenna geometry gives converged results for both near and far field parameters. In the context of electrically large platforms it was found that background meshing sampling can be relaxed to $\lambda / 10$ and $\lambda / 8$ without significantly compromising the accuracy of results. This is an important result that shows significant improvement in computational efficiency when compared to the minimum requirement of the FDTD method of $\lambda / 20$ [2] and is similar to that of the MLFMM method [1]. For large platform sizes truncating the computational box to just below the ground plane yields additional computational savings without compromising the accuracy of results. Finally, when the antenna is installed in an aircraft wing it was shown that the near-field interactions between the antenna and the wing platform are more disruptive than when antenna is positioned on a flat platform. The radome cover causes additional disruption to the antenna pattern by severely reducing radiation intensity in the main beam and increasing the radiation in the sidelobes.

\section{ACKNOWLEDGMENT}

The authors wish to thank Dr S. Earl and Prof. C. Jones of BAE SYSTEMS for many valuable discussions.

\section{REFERENCES}

[1] M. V. T. Heckler, A. Dreher, "Performance of microstrip antenna arrays installed on aircraft", Aerospace Science and Technology, vol.26, pp.235243, 2013.

[2] S.P. Benham, J. A. Lord, T. J. Murphy, D.J. Knights, "Application of AGATE (A Finite Difference Time Domain Tool) to antennas on complex platforms", The $2^{\text {nd }}$ Conference on Antennas and Propagation, EuCAP, DOI: 10 1049/ic.2007.1151, Nov 2007.

[3] I. Wood, "Transient analysis of installed antenna performance", Antenna and Propagation Society International Symposium (APSURSI), DOI: 10.1109/APS.2010.5561126, 2010.

[4] S, -P.Gao, B. Wang, H. Zhao, W.-J. Zhao, C. E. Png, "Installed radiation pattern of patch antennas", IEEE Antennas and propagation magazine, pp.81-94, June 2015.
[5] A. Barka, "Domain decomposition method based on generalised scattering matrix for installed performance of antennas on aircraft", IEEE Trans. Antennas Propag., vol. 55, pp.1833-1842, June 2007.

[6] A. Barka, "Integration of antennas on board vehicles and diffraction by large and complex structures with multiple-domain-multiple-methods techniques", Proc. IEEE, Vol.101, pp.280-297, Feb 2013.

[7] F Weinmann, "Ray Tracing with PO/PTD for RCS modelling of large complex objects", IEEE Trans. Antennas Propagat., Vol.54, pp.1797$1806,2006$.

[8] R. U. Nair and R. M. Jha, "Electromagnetic design and performance analysis of airborne radomes trends and perspectives", IEEE Antennas and Prop. Magazine, vol. 56, pp. 276-298, 2014.

[9] X. Wang, Z. Peng, K.-H. Lim, J.-F. Lee, "Multisolver Domain Decomposition Method for Modeling EMC Effects of Multiple Antennas on a Large Air Platform", IEEE Trans. Antennas Propagat., vol. 54, no. 2, pp. 375-88, 2012.

[10] Z-L. Liu, X. Wang, C-F. Wang, "Installed Performance Modeling of Complex Antenna Array Mounted on Extremely Large-Scale Platform Using Fast MoM-PO Hybrid Framework", IEEE Trans. Antennas Propagat., vol. 62, no. 7, pp. 3852-8, 2014.

[11] Ilcev St. D., "Airborne Satellite Navigation and Other Integrated Antenna Systems", International Conference on Electrical, Electronics, and Optimization Techniques (ICEEOT), 2016, pp. 4493-4500.

[12] J. Alvarez, "Simplified antenna models for the prediction of installed performances on large platforms" Proc. Antenna Measurements \& Applications (CAMA), 16-19 Nov. 2014.

[13] B. Le Lepvrier, R. Loison, R. Gillard, P. Pouliguen, P. Potier, L.Patier "A New Hybrid Method for the Analysis of Surrounded Antennas Mounted on Large Platforms", IEEE Trans. Antennas Propagat., vol. 62, no. 5, pp. 2388-97, 2014.

[14] W-J. Zhao, L.-W. Li Y.-B. Gan "Efficient Analysis of Antenna Radiation in the Presence of Airborne Dielectric Radomes of Arbitrary Shape IEEE Trans. Antennas Propagat., vol. 53, no. 1, pp. 442-9, 2005.

[15] S. Rappaport, S. Sun, R. Mayzus, H. Zhao, Y. Azar, K. Wang, G. N. Wong, J. K. Schulz, M. Samimi, F. Gutierrez, "Millimeter wave Mobile Communications for 5G Cellular: it will work!", IEEE Access, 2013.

[16] R. Mittra, "A Look at some challenging problems in computational electromagnetics", IEEE Trans. Antennas Propagat., vol.46, pp.18-32, 2004.

[17] R. A. Martin, D. H. Werner, "A reciprocity approach for calculating the far-field radiation patterns of a helical microstrip antenna mounted on a dielectric-coated circular cylinder", IEEE Trans. Antennas Propagat., vol.49, pp.1754-1762, Dec.2001

[18] D. H. Werner, R. J. Allard, R. A. Martin, R. Mittra, "A reciprocity approach for calculating radiation patterns of arbitrarily shaped microstrip antennas", IEEE Trans. Antennas Propagat., vol.51, pp.730737, April 2003

[19] D Jiao, A. A. Ergin, B. Shanker, E. Michielssen, J. -M. Jin, "A fast timedomain finite element-boundary integral method for electromagnetic analysis", IEEE Trans. Antennas Propagat., vol.49, pp.1453-1461, Oct. 2001.

[20] P. Lalande, A. Delannoy, "Numerical Methods for Zoning Computation", AerospaceLab, 2012, issue 5, Dec. 2012

[21] J.P. Parmantier, F. Issac, V. Gobin. "Indirect Effects of Lightning on Aircraft and Rotorcraft", AerospaceLab issue 5, Dec. 2012.

[22] T. K. Sarkar, A. R. Djordjevic, B. M. Kolundzija, "Method of moments applied to antennas" in Chapter 8 of Handbook of Antennas in Wireless Communications, CRC Press, 2001.

[23] Jian-Ming Jin, The Finite Element Method in Electromagnetics, 2nd ed. New York: John Wiley \& Sons, 2002.

[24] A. Taflove and S. C. Hagness, Computational Electrodynamics: The Finite-Difference Time-Domain Method, 2nd ed. Norwell, MA: Artech House, 2000.

[25] C. Christopoulos, The Transmission line modelling method: TLM, IEEE Press, New York, NY, 1995.

[26] P. Russer, The Transmission Line Matrix Method, in Applied Computational Electromagnetics, ser. NATO ASI. London, U.K.: Springer-Verlag, 2000, pp. 243-269.

[27] P. Sewell, T. M. Benson, C. Christopoulos, D. W. P. Thomas, A Vukovic, J G. Wykes, "Transmission line modelling (TLM) based upon unstructured tetrahedral meshes", IEEE Trans. Microwave Theory and Tech. vol. 53, pp.1919-1928, June, 2005. 
3

[28] P. Sewell, A. Vukovic, X. Meng, R. M. Benson, "A note on material losses in unstructured transmission line modelling", Microwave and Optical Techn. Letters, vol.57, pp.2218-2222, September, 2015.

[29] X. Meng, P. Sewell, S. Phang, A. Vukovic, T. M. Benson, "Modelling curved carbon fiber composite (CFC) structures in the Transmission-Line Modelling (TLM) Method", IEEE Trans. on EMC, vol.57, pp.384-390, June 2015

[30] P. Sewell, T.M. Benson, C. Christopoulos, D. W. P. Thomas, A. Vukovic, J. G. Wykes, Implicit element clustering for tetrahedral transmission-line modelling (TLM), IEEE Trans. Microw. Theory Tech., vol.57, pp. $2005-$ 2014, 2009.

[31] X. Meng, P. Sewell, N. H. A. Rahman, A. Vukovic, T.M. Benson, "Experimental benchmarking of unstructured transmission line modelling (UTLM) method in modelling twisted wires", ACES Express Journal,vol.1, pp. 101-104, 2016.

[32] X Meng, A. Vukovic, T. M. Benson, P. Sewell, "Extended capability models for carbon fiber composite(CFC) panels in the unstructured transmission line modelling (UTLM) method", IEEE Trans. Electromagnetic Compat., vol.58, pp. 811-819, 2016.

[33] P. Sewell, A. Vukovic, T. M. Benson, X. Meng, Extracting Modal Field Profiles from 3D Unstructured TLM Meshes for use as Sources and Observers, IET Science, Measurements \& Technology, DOI: 10.1049 /iet-smt.2016.0357 April 2017

[34] P. Sewell, T.M. Benson, A. Vukovic, X. Meng, "Complexity Reduction of Multiscale UTLM Cell Clusters", IEEE J. on Multiscale and Multiphysics Computational Techniques, vol.2, pp.18-28, 2017.

[35] C. Jones, P. Sewell, T. M. Benson, A. Vukovic, X. Meng and H.Bucklow: "Advanced computational electromagnetics at BAE systems", Proc. NAFEMS UK Conf., Telford, UK, pp. 1-4, June 2016.

[36] A. Bossavit, "Yee-like schemes on a tetrahedral mesh, with diagonal lumping”, Int. J. Numer. Modeling, vol. 12, no. 1-2, pp. 129-142, 1999.

[37] J. Keranen, J. Kangas, A. Ahola and L. Kettunen, "Implicit Yee-like scheme on tetrahedral Mesh", IEEE Trans. on Magnetics, vol. 38, no. 2, pp. 717-720, 2002.

[38] J. Gao, G. Kobidze and B. Shanker, "Higher-order FDTD algorithm using tetrahedral Tessellation", Proc. IEEE AP-S Int. Antennas and Propagation Symp, vol. 4, Columbus, USA, pp. 364 - 367, Jun. 2003,

[39] S.-W. Cheng, T. K. Dey, J. Shewchuk, Delaunay mesh generation, Chapman \& Hall/CRC Computer \& Information Science Series, 2012.

[40] https://www.nottingham.ac.uk/research/groups/ggiemr/

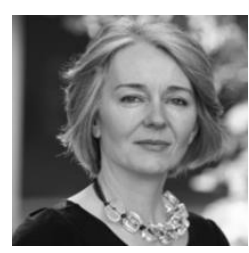

Ana Vukovic (M'97) was born in Nis, Yugoslavia, in 1968. She received the Diploma of Engineering degree in electronics and telecommunications from the University of Nis, Nis, Yugoslavia, in 1992, and the Ph.D. degree from the University of Nottingham, Nottingham, U.K., in 2000.

From 1992 to 2001, she was a Research Associate with the University of Nottingham. In 2001, she joined the School of Electrical and Electronic Engineering, University of Nottingham, as a Lecturer. Her research interests are electromagnetics with a particular emphasis on applications in optoelectronics, microwaves, and EMC.

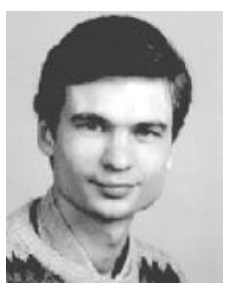

Phillip Sewell (M'89-SM'04) was born in London, U.K., in 1965. He received the B.Sc. degree in electrical and electronic engineering (with first-class honors) and Ph.D. degree from the University of Bath, Bath, U.K., in 1988 and 1991, respectively.

From 1991 to 1993, he was a Post-Doctoral Fellow with the University of Ancona, Ancona, Italy. In 1993 , he became a Lecturer with the School of Electrical and Electronic Engineering, University of Nottingham, Nottingham, U.K. In 2001 and 2005, he became a Reader and Professor of electromagnetics at the University of Nottingham. His research interests involve analytical and numerical modeling of electromagnetic problems with application to opto-electronics, microwaves and aerospace applications.

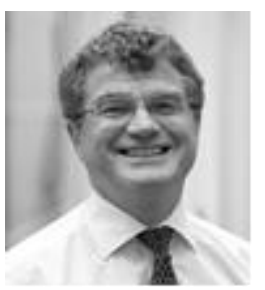

Trevor M. Benson received a First Class honors degree in Physics and the Clark Prize in Experimental Physics from the University of Sheffield in 1979, a PhD in Electronic and Electrical Engineering from the same University in 1982 and the DSc degree from the University of Nottingham in 2005 .

After spending over six years as a Lecturer at University College Cardiff, Professor Benson moved to The University of Nottingham in 1989. He was promoted to a Chair in Optoelectronics in 1996, having previously been Senior Lecturer (1989) and Reader (1994). Professor Benson's research interests include experimental and numerical studies of electromagnetic fields and waves with particular emphasis on the theory, modeling and simulation of optical waveguides, lasers and amplifiers, nano-scale photonic circuits and electromagnetic compatibility.

$\mathrm{He}$ is a Fellow of the Institute of Engineering Technology (FIET) and the Institute of Physics (FInst.P). He was elected a Fellow of the Royal Academy of Engineering in 2005 for his achievements in the development of versatile design software used to analyze propagation in optoelectronic waveguides and photonic integrated circuits. 